\title{
Kaposi's Sarcoma of the Bulbar Conjunctiva in an Immunocompetent Patient
}

\author{
Dimitris Mikropoulos ${ }^{a}$ Ioannis Mavrikakis ${ }^{b}$ \\ Nikolas G. Ziakas ${ }^{\text {a }}$ Anastasios G.P. Konstas ${ }^{a}$ \\ Kostas G. Boboridis ${ }^{a}$ \\ aFirst Ophthalmology Department, Aristotle University of Thessaloniki, \\ Thessaloniki, and ' Oculoplastic Department, Metropolitan Hospital, \\ Athens, Greece
}

\section{Key Words}

Kaposi's sarcoma · Conjunctiva · Tumor · AIDS - Immunocompetent · Immunosuppression

\begin{abstract}
Kaposi's sarcoma is an uncommon endothelial malignant tumor, first described by Moricz Kaposi in 1872. It is commonly encountered in patients with acquired immunodeficiency syndrome (AIDS), immunosuppression or organ transplantation. Conjunctival Kaposi's sarcoma is a rare tumor, mostly in patients with AIDS. We present a selected case with a superior bulbar conjunctival lesion in a patient who is HIV negative and immunocompetent, with an unremarkable general medical history. The lesion was surgically excised with no adjunctive treatment, and histological examination confirmed the diagnosis. There was no recurrence or metastasis 5 years after surgery.
\end{abstract}

\section{Introduction}

Kaposi's sarcoma (KS) is an uncommon spindle cell malignancy of endothelial cell origin, first described by Moricz Kaposi in 1872. It is frequently encountered in patients with acquired immunodeficiency syndrome (AIDS), immunosuppression, organ transplantation or human herpes simplex virus-8 (HHV-8) infection [1-3]. Conjunctival sarcoma is rare and is encountered mostly in patients with AIDS, in some cases as the first clinical presentation $[4,5]$. We report a case of an immunocompetent patient with a superior bulbar conjunctival KS, managed successfully with surgical excision alone. 


\section{Case Report}

A 70-year-old male presented with a 3-month history of a gradually growing mass on his left superior bulbar conjunctiva ( $\underline{\text { fig. } 1 \mathrm{la}}$ ). Examination revealed a dark red, firm, painless mass with a thinner stalk attachment on the conjunctiva and no evidence of orbital or scleral invasion (fig. 1b). This was an isolated ocular involvement in a HIV-negative patient, with no clinical evidence of immunosuppression and unremarkable general health.

We applied our standard protocol for a potentially malignant conjunctival lesion. Surgical excision included a 2-mm margin of healthy tissue around the conjunctival stalk and the underlying Tenon's capsule followed by meticulous hemostasis with a bipolar cautery. Additional intervention like cryotherapy or radiotherapy was reserved for a possible incomplete excision depending on the histological diagnosis. The bare sclera defect was allowed to granulate with secondary intention healing. Conjunctival surface reconstruction was avoided in order to monitor the surgical site for possible recurrence of the tumor. The bulbar conjunctiva healed with a smooth surface, no signs of

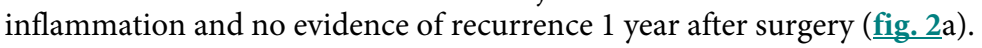

Histological examination confirmed the diagnosis of a stage $b$ and $c$ KS, characterized by the presence of spindle cells positive on CD31, CD34 and Ulex Europaeus markers with minimal pleomorphism and mitotic activity. They are forming slits containing extravasated red blood cells, whereas extracellular or intracellular periodic acid-Schiff-positive hyaline bodies can also be found [6] (fig. 2b). Histology revealed that the tumor was excised with a clear margin of healthy conjunctival tissue with no deep invasion to the underlying tissues. The specimen was also tested negative for HHV-8 infection by means of polymerase chain reaction (PCR). In view of the special clinical characteristics of such a malignancy, the patient was referred to the immunology and infectious diseases department for evaluation and subsequent postoperative follow-up of his general health. He was diagnosed immunocompetent with normal immunoglobulin levels and no evidence of HIV or other viral infection. His immune condition remained unremarkable throughout the observation period of 5 years.

\section{Discussion}

Ocular KS is rare in HIV-negative patients with less than 30 cases being reported before 1982. Only $20 \%$ of AIDS patients suffering from KS have ocular manifestations (lids or conjunctiva) and in $12 \%$ of cases ocular involvement is the primary manifestation of systemic disease [7-9].

Ocular lesions have slow growth and can be cured with simple surgical excision whereas cryotherapy is an adjunctive intervention which was not used in our case because of the clear tumor margins and the lack of systemic involvement. Radiotherapy with chemotherapy is reserved for multifocal aggressive disease [10-12].

Simple complete surgical excision was curative in our case with no evidence of recurrence or distant metastasis and no immune-related general health problems in the postoperative observation of 5 years. Differential diagnosis of similar lesions should include arteriovenous shunt, pyogenic granuloma, malignant blue nevus, bacterial angiomatosis and vascular malformations. Isolated KSs of the conjunctiva unrelated to AIDS have been reported previously [13].

This case may strengthen the evidence that such conjunctival malignancies may occur as an isolated incidence and do not necessarily relate to AIDS or immunosuppression [14]. Simple surgical excision of the conjunctival lesion, with no adjunctive measure, can be curative with no evidence of recurrence or distant metastasis in a long-term follow-up of 5 years. 


\section{Disclosure Statement}

The authors have no proprietary interest and received no financial support in relation to this manuscript.

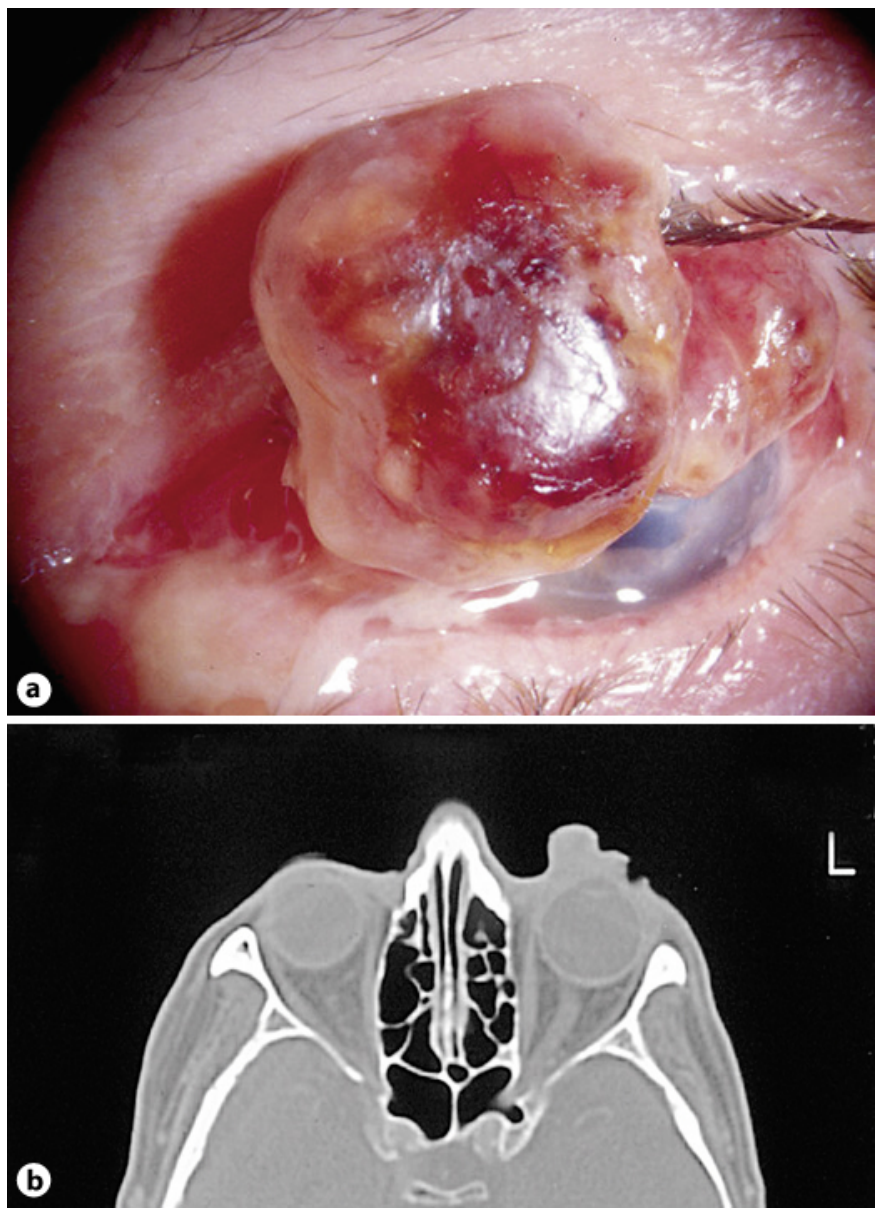

Fig. 1. a Solid mass on the superior bulbar conjunctiva displacing the upper eyelid upwards. b CT scan imaging of the orbits shows no scleral or orbital extension of the lesion. 


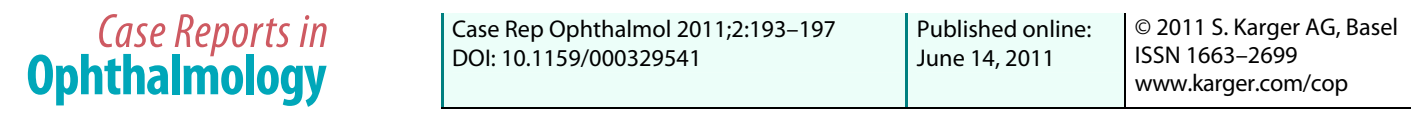
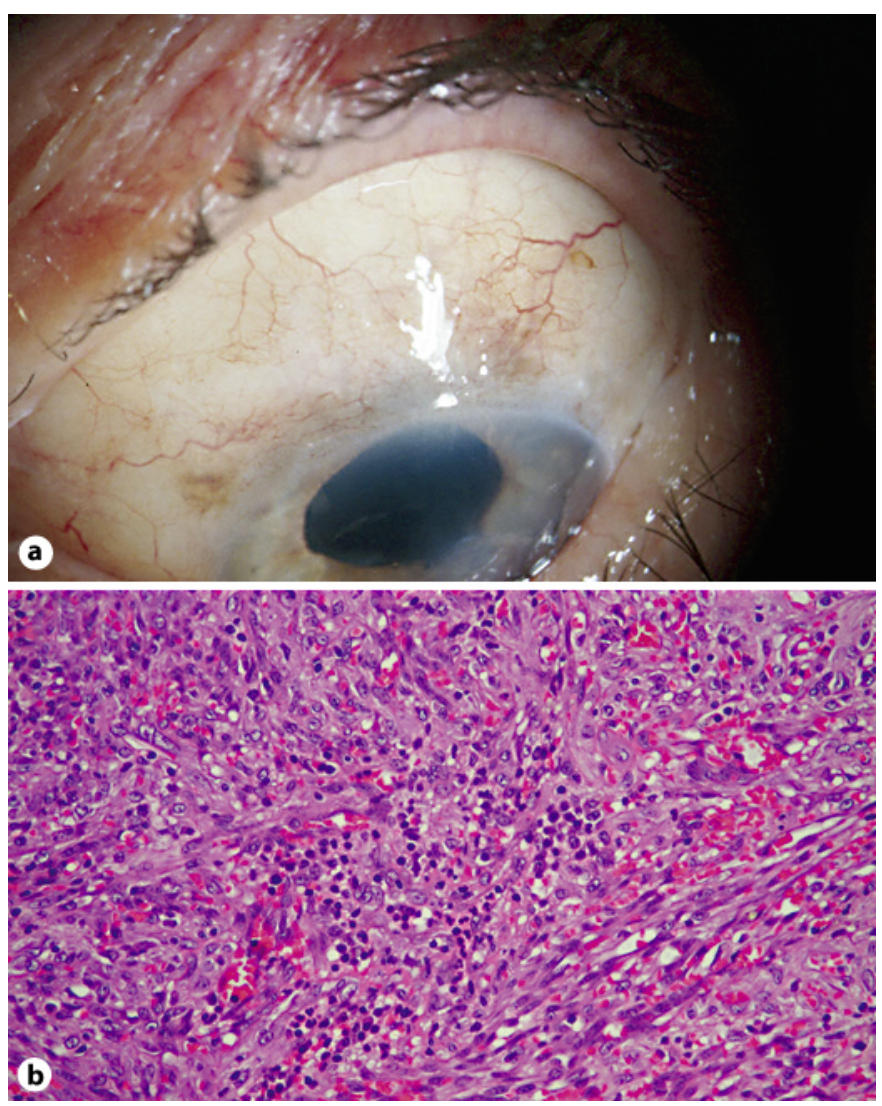

Fig. 2. a Appearance of the bulbar conjunctiva 12 months after surgery with little visible scar tissue and no evidence of recurrence. $\mathbf{b}$ Histology section shows the characteristic neoplastic spindle cells forming slits filled with extravasated red blood cells. HE, $\times 200$.

\section{References}

$\checkmark 1$ Verma V, Shen D, Sieving PC, Chan CC: The role of infectious agents in the etiology of ocular adnexal neoplasia. Surv Ophthalmol 2008;53:312-331.

2 Howard GM, Jakobiec FA, DeVoe AG: Kaposi's sarcoma of the conjunctiva. Am J Ophthalmol 1975;79:420423.

-3 Lieberman PH, Llovera IN: Kaposi's sarcoma of the bulbar conjunctiva. Arch Ophthalmol 1972;88:44-45.

4 Curtis TH, Durairaj VD: Conjunctival Kaposi sarcoma as the initial presentation of human immunodeficiency virus infection. Ophthal Plast Reconstr Surg 2005;21:314-315.

5 Kurumety UR, Lustbader JM: Kaposi's sarcoma of the bulbar conjunctiva as an initial clinical manifestation of acquired immunodeficiency syndrome. Arch Ophthalmol 1995;113:978.

6 Weiter JJ, Jakobiec FA, Iwamoto T: The clinical and morphologic characteristics of Kaposi's sarcomas of the conjunctiva. Am J Ophthalmol 1980;89:546-552.

7 Schmid K, Wild T, Bolz M, Horvat R, Jurecka W, Zehetmayer M: Kaposi's sarcoma of the conjunctiva leads to a diagnosis of acquired immunodeficiency syndrome. Acta Ophthalmol Scand 2003;81:411-413.

-8 Shuler JD, Holland GN, Miles SA, Miller BJ, Grossman I: Kaposi sarcoma of the conjunctiva and eyelids associated with the acquired immunodeficiency syndrome. Arch Ophthalmol 1989;107:858-862.

-9 Kalinske M, Leone CR Jr: Kaposi’s sarcoma involving eyelid and conjunctiva. Ann Ophthalmol 1982;14:497499. 
10 Kirova YM, Belembaogo E, Frikha H, Haddad E, Calitchi E, Levy E, Piedbois P, Le Bourgeois JP: Radiotherapy in the management of epidemic Kaposi's sarcoma: a retrospective study of 643 cases. Radiother Oncol $1998 ; 46: 19-22$.

11 Ghabrial R, Quivey JM, Dunn JP Jr, Char DH: Radiation therapy of acquired immunodeficiency syndromerelated Kaposi's sarcoma of the eyelids and conjunctiva. Arch Ophthalmol 1992;110:1423-1426.

-12 Dugel PU, Gill PS, Frangieh GT, Rao NA: Treatment of ocular adnexal Kaposi's sarcoma in acquired immune deficiency syndrome. Ophthalmology 1992;99:1127-1132.

13 Ron IG, Kremer I, Lowenstein A, Chaitchik S: Conjunctival involvement in classic (indolent) HIV negative Kaposi's sarcoma. Br J Ophthalmol 1994;78:488-489.

14 Reiser BJ, Mok A, Kukes G, Kim JW: Non-AIDS-related Kaposi sarcoma involving the tarsal conjunctiva and eyelid margin. Arch Ophthalmol 2007;125:838-840.

This case report was first presented at the 20th meeting of the European Society of Ophthalmic Plastic and Reconstructive Surgery (ESOPRS), Munster, Germany, September 2002. 